\title{
Reliability assessment of heritage architecture
}

\author{
M. Holicky \& M. Sykora \\ Klokner Institute, Czech Technical University in Prague, Czech Republic
}

\begin{abstract}
Reliability assessment of heritage structures is in many aspects different from structural design. The effects of the construction process and subsequent life of the architecture, during which it may have undergone alteration, deterioration or misuse must be taken into account. That is why the assessment of heritage architecture often requires application of sophisticated methods, as a rule beyond the scope of design codes. The two main principles for the assessment may be summarized as follows: (1) Available scientific knowledge and experience including currently valid codes should be applied; (2) Actual characteristics of structural materials, actions, geometric data and structural behaviour should be considered. The most important step of the whole assessment may be evaluation of inspection data.
\end{abstract}

Keywords: structures, assessment, heritage architecture, reliability.

\section{Introduction}

The most developed societies perceive necessary to maintain architectural heritage [1]. However, the need for rehabilitations of heritage structures is often confined by severe economic constraints. Structural strengthening is then considered as the most sensitive aspect of the rehabilitations since it may conflict with the heritage value. That is why assessment of heritage structures often requires application of sophisticated methods, as a rule beyond the scope of traditional design codes. Nevertheless, apart from few national codes, three international standards ISO [2-4] related to assessment of existing structures are available. ISO 13822 [3] contains an annex focused on heritage structures. Additional information may be found in a number of scientific papers and publications such as $[5,6]$.

In general a heritage structure may be subjected to the reliability assessment in the case of: 
- Rehabilitation during which new structural members are added to an existing load-carrying system;

- Adequacy checking in order to establish whether the heritage structure can resist loads associated with the anticipated change in use, operational changes or extension of its working life;

- Repair of a heritage structure, which has deteriorated due to time dependent environmental effects or which has suffered damage from accidental actions, for example earthquake;

- Doubts concerning actual reliability of the structure.

Under some circumstances assessments may also be required by authorities, insurance companies or owners or may be demanded by a maintenance plan.

The approach to assessment of a heritage structure is in many aspects different from that taken in structural designing. Difficulties in assessments of heritage structures may arise from the complexity of geometry, variability of material properties, different construction techniques, limited knowledge on structural conditions including the damage from past actions, and from interventions restricted by heritage value and excessive costs [7]. Methods of experimental mechanics and numerical simulation approach have been rather recently introduced in professions which had been for long reserved only for humanities or arts. Conservation of cultural heritage belongs among such fields.

However, even though the heritage structure may be investigated several times, some uncertainty in the basic variables and structural behaviour shall always remain. Therefore, similarly as in design of new structures, actual variation in the basic variables describing actions, material properties, geometric data and model uncertainties are taken into account by partial factors or other code provisions.

The paper summarises general principles of the structural assessment of heritage structures. The term "heritage structures" covers monumental historical buildings such as castles and churches, and also bridges, industrial heritage structures as well as master-pieces of modern architecture.

\section{Principles of assessment}

Two main principles are usually accepted when assessing heritage structures:

(1) Currently valid codes for verification of structural reliability should be applied; historic codes valid in the period of structural design should be used only as guidance documents.

(2) Actual characteristics of structural materials, actions, geometric data and structural behaviour should be considered, the original design documentation including drawings should be used as guidance only.

The first principle should be applied in order to achieve a similar reliability level as in case of newly designed structures. The second principle should avoid negligence of any structural condition that may affect actual reliability (in a favourable or unfavourable way) of the structure. 
Most of the current codes have been developed assuming the concept of limit states in conjunction with the partial factor method. In accordance with this method, which is mostly considered here, basic variables are specified by characteristic or representative values. The design values of basic variables are determined on the basis of the characteristic (representative) values and appropriate partial factors.

It follows from the second principle that a visual inspection of the assessed structure should be made whenever possible. Practical experience shows that inspection of the site is also useful to obtain a good feel for actual situation and state of the structure.

As a rule quantitative assessments need not to be performed for those parts of the structure that will not be affected by structural changes, rehabilitation, repair, change in use or which are not obviously damaged or not suspected of having insufficient reliability, ISO 13822 [3]. In general the assessment procedure consists of the following steps (see the flow chart in [3]):

- Specification of the assessment objectives required by a client or authority;

- Scenarios related to structural conditions and actions;

- Preliminary assessment: study of available documentation, preliminary inspection, preliminary checks, decision on immediate actions and recommendation for detailed assessment;

- Detailed assessment: detailed documentary search, detailed inspection, material testing and determination of actions, determination of structural properties, structural analysis, verification of structural reliability;

- Report including proposal for construction intervention;

- Repeat the sequence if necessary.

When the preliminary assessment indicates that the structure is reliable for its intended use over the remaining life, a detailed assessment may not be required. Conversely if the structure seems to be in dangerous or uncertain conditions immediate interventions and detailed assessment may be necessary.

In accordance with Annex I of ISO 13822 [3] structural assessment of a heritage structure should be carried out in collaboration with a multidisciplinary team of engineers, architects, archaeologists, historians, material scientists and possibly other specialists. While the structural engineer should deliver a specific structural evaluation report, the ramifications of this report should be discussed within the multi-disciplinary team and decisions should be generally reached by consensus.

\section{Investigation}

Investigation of a heritage structure is intended to verify and update the knowledge about the present condition (state) of the structure with respect to a number of aspects. Often, the first impression of the structural condition will be based on visual qualitative investigation. The description of possible damage of the structure may be presented in verbal terms like: 'unknown, none, minor, 
moderate, severe, destructive'. Very often the decision based on such an observation will be made by experts in a purely intuitive way.

A better judgement of the structural condition can be made on the basis of (subsequent) quantitative inspections. Typically, the assessment is a cyclic process when the first inspection is supplemented by subsequent investigations. The purpose of the subsequent investigations is to obtain a better feel for the actual structural condition (particularly in the case of damage) and to verify information required for determination of the characteristic and representative values of all basic variables. For all inspection techniques, information on the probability of detecting damage if present, and the accuracy of the results should be given.

The statement from the investigation contains, as a rule, the following data describing:

- Actual state of the structure;

- Types of structural materials and soils;

- Observed damage;

- Actions including environmental effects;

- Available design documentation.

Proof loading is a special type of investigation. Based on such tests one may draw conclusions with respect to:

- The bearing capacity of the tested member under the test load condition;

- Other members;

- Other load conditions;

- The behaviour of the system.

The inference in the first case is relatively easy; the probability density function of the load bearing capacity is simply cut off at the value of the proof load. The inference from the other cases is more complex. Note that the number of proof load tests needs not to be restricted to one. Proof testing may concern one element under various loading conditions and/or a sample of structural elements. In order to avoid unnecessary damage to the structure due to the proof load, it is recommended to increase the load gradually and to measure the deformations. Measurements may also give a better insight into the behaviour of the system. In general, proof loads can hardly address long-term or timedependent effects. These effects should be analysed by calculation.

\section{Basic variables}

In accordance with the above-mentioned general principles, characteristic and representative values of all basic variables shall be determined taking into account the actual situation and state of the structure. Available design documentation is used as a guidance material only. Actual state of the structure should be verified by its inspection to an adequate extent. If possible non- 
destructive or minor destructive tests should be preferred; results should be evaluated by statistical methods taking into account measurement uncertainty.

For reliability verification using the partial factor method, the characteristic and representative values of basic variables shall be considered as follows:

(1) Dimensions of the structural elements shall be determined on the basis of adequate measurements. However, when the original design documentation is available and no changes in dimensions have taken place, the nominal dimensions given in the documentation may be used in the analysis.

(2) Load characteristics shall be introduced with the values corresponding with the actual situation verified by destructive or non-destructive inspections. When some loads have been reduced or removed completely, the representative values can be reduced or appropriate partial factors can be adjusted. When overloading has been observed in the past it may be appropriate to increase adequately representative values.

(3) Material properties shall be considered according to the actual state of the structure verified by destructive or non-destructive inspections. When the original design documentation is available and no serious deterioration, design errors or construction errors are suspected, the characteristic values given in original design may be used.

(4) Model uncertainties shall be considered in the same way as in design stage unless previous structural behaviour (especially damage) indicates otherwise. In some cases model factors, coefficients and other design assumptions may be established from measurements on the heritage structure (e.g. wind pressure coefficient, effective width values etc.).

Thus the reliability verification should be backed up by inspection of the structure including collection of appropriate data. Evaluation of prior information and its updating using newly obtained measurements is one of the most important steps of the assessment.

\section{Evaluation of inspection results}

Using results of an investigation (qualitative inspection, calculations, quantitative inspection, proof loading) the properties and estimates of structural reliability may be updated. Two different procedures can be distinguished:

(1) Updating of the structural failure probability.

(2) Updating of the probability distributions of basic variables.

Direct updating of the structural reliability (procedure (1)) can be formally carried out using the following basic formula of the probability theory:

$$
\mathrm{P}(F \mid I)=\mathrm{P}(F \cap I) / \mathrm{P}(I)
$$


where $\mathrm{P}$ denotes probability; $F$ local or global failure; $I$ inspection information; and $\cap$ the intersection of two events. The inspection information $I$ may consist of survival of a significant overloading; material characteristics from different sources; known geometry, damage and deterioration; outcome of visual inspections etc. More details and numerical examples are provided elsewhere [8].

The updating procedure of a univariate or multivariate probability distribution (procedure (2)) is given formally as:

$$
\mathrm{f}_{X}(x \mid I)=C \mathrm{P}(I \mid x) \mathrm{f}_{X}(x)
$$

where $X$ denotes a basic variable or statistical parameter; $\mathrm{f}_{X}(x \mid I)$ updated probability density function of $X ; \mathrm{f}_{X}(x)$ probability density function of $X$ before updating; $C$ normalising constant; and $\mathrm{P}(I \mid x)$ is a likelihood function.

An illustration of Equation (2) is presented in Figure 1. In this example updating leads to a more favourable distribution with a greater design value $x_{\mathrm{d}}$ than the prior design value. In general, however, the updated distribution might be also less favourable than the prior distribution.

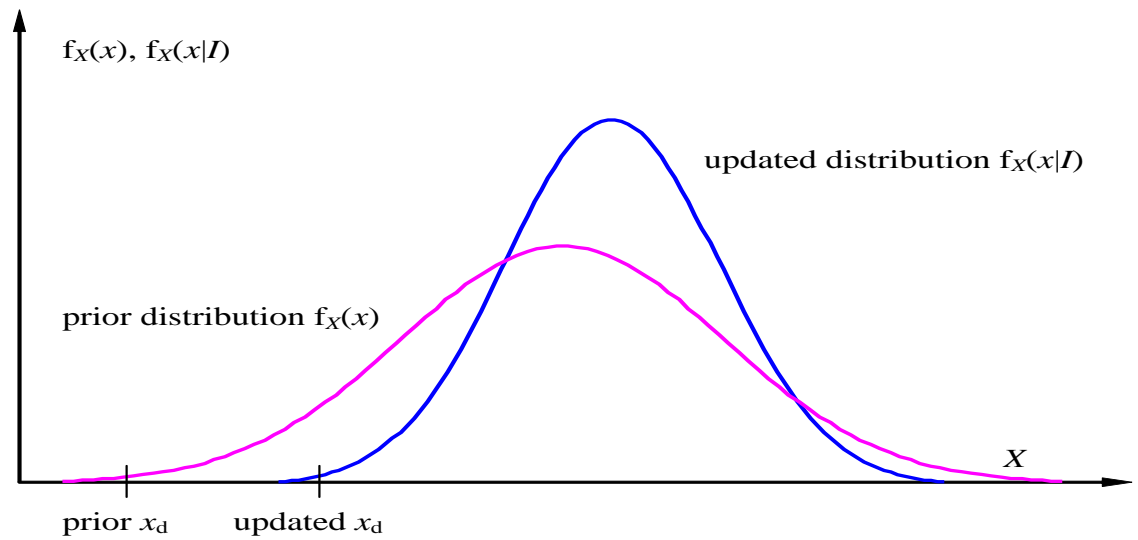

Figure 1: $\quad$ Updating of probability density function for a basic variable $X$.

The updating procedure can be used to derive updated characteristic and representative values (fractiles of appropriate distributions) of basic variables to be used in the partial factor method or to compare directly action effects with limit values (cracks, displacements). More information on updating may be found in ISO 12491 [4] or in the monograph [9].

Once the updated distributions for the basic variables $f_{X}(x)$ have been found, the updated failure probability $\mathrm{P}(F \mid I)$ may be determined by performing a probabilistic analysis using common method of structural reliability for new structures. Symbolically it can be written:

$$
\mathrm{P}(F \mid I)=\int_{\mathrm{G}(X)<0} \mathrm{f}_{X}(x \mid I) \mathrm{d} x
$$


where $\mathrm{G}(X)<0$ denotes the failure domain $(\mathrm{G}(X)$ being the limit state function). It should be proved that the probability $\mathrm{P}(F \mid I)$, given the updated distributions of basic variables, does not exceed a specified target value.

A more practical procedure is to determine updated design values for each basic variable (procedure (2)). For a resistance parameter $X$, the design value can be obtained using operational formula provided by ISO 2394 [2]. For normal and lognormal random variable it holds, respectively:

$$
x_{\mathrm{d}}=\mu(1-\alpha \beta V) ; \quad x_{\mathrm{d}}=\mu \exp \left(-\alpha \beta \sigma-0.5 \sigma^{2}\right)
$$

where $\mu$ is the updated mean value; $\alpha$ FORM (First Order Reliability Method) sensitivity factor; $\beta$ target reliability index; $V$ updated coefficient of variation; and $\sigma^{2}=\ln \left(1+V^{2}\right)$. The target reliability index $\beta$ is discussed in the next section, the value of $\alpha$ can be taken in accordance with ISO 2394 [2] as follows: 0.8 and 0.32 for a dominating and non-dominating resistance parameter; and 0.7 and 0.28 for dominating and non-dominating load parameter.

Note that the coefficient of variation may cover variability of the basic variable (coefficient of variation $V_{X}$ ), and also effects of the uncertainty in a considered resistance model $\left(V_{\theta}\right)$, variability of geometry $\left(V_{\text {geo }}\right)$ and measurement errors $\left(V_{\varepsilon}\right)$. In common cases the following relationship provides a good approximation:

$$
V \approx \sqrt{ }\left(V_{X}^{2}+V_{\theta}^{2}+V_{\mathrm{geo}}^{2}+V_{\varepsilon}^{2}\right)
$$

In the case of a biased model uncertainty or measurement error, also the mean $\mu$ needs to be modified accordingly.

As an alternative to procedure (2), one might also determine the characteristic value $x_{\mathrm{k}}$ first and calculate the design value $x_{\mathrm{d}}$ by applying the appropriate partial factor $\gamma_{\mathrm{m}}$ :

$$
x_{\mathrm{d}}=x_{\mathrm{k}} / \gamma_{\mathrm{m}}
$$

For normal and lognormal random variable $X$ the characteristic value $x_{\mathrm{k}}$ then follows as:

$$
x_{\mathrm{k}}=\mu(1-k V) ; \quad x_{\mathrm{k}}=\mu \exp \left(-k \sigma-0.5 \sigma^{2}\right)
$$

where $k=1.64$ is the $5 \%$ fractile of the standardised normal distribution. It may be helpful to consider both methods and to use a more conservative result. This procedure may be applied for all basic variables. However, for geomechanical properties and variable loads usually other distributions apart from the normal and lognormal distribution are more suitable.

Note that a lower acceptable reliability level can be specified by reducing $\beta$ values for probabilistic design and reducing $\gamma$-values in the partial factor method. For a material property $X$ described by a normal distribution the partial factor $\gamma_{\mathrm{m}}$ may be estimated using equation: 


$$
\gamma_{\mathrm{m}}=x_{\mathrm{k}} / x_{\mathrm{d}}=(1-k V) /(1-\alpha \beta V)
$$

Similar relationships may be derived for lognormal or other distributions [10].

\section{Structural analysis and verification}

Structural behaviour should be analysed using models that describe actual situation and state of a heritage structure. Generally the structure should be analysed for ultimate and serviceability limit states using basic variables and considering relevant deterioration processes.

All basic variables describing actions, material properties, load and model uncertainties should be considered as mentioned above. The uncertainty associated with the validity and accuracy of the models should be considered during assessment, either by adopting appropriate factors in deterministic verifications or by introducing probabilistic model factors in reliability analysis.

When a structure is analysed, conversion factors reflecting the influence of shape and size effect of specimens, temperature, moisture, duration-of-load effect etc., should be taken into account. An example is minor destructive drilling of masonry units. The level of knowledge about the condition of components should be also considered. This can be achieved by adjusting the assumed variability in either the load carrying capacity of the components or the dimensions of their cross sections, depending on the type of structure.

When deterioration is observed, the relevant mechanisms shall be identified and a deterioration model predicting the future performance of the structure shall be determined on the basis of theoretical or experimental investigation, inspection and experience. Even considerably simplifying assumptions may yield realistic results as shown for degradation of reinforced concrete structures in [11].

Reliability verification of a heritage structure shall be made using valid codes of practice, as a rule based on the limit state concept. Attention should be paid to both the ultimate and serviceability limit states. Verification may be carried out using partial safety factor or structural reliability methods with consideration of structural system and ductility of components. The reliability assessment shall be made taking into account the remaining working life of a structure, the reference period, and changes in the environment of a structure associated with an anticipated change in use.

The conclusion from the assessment shall withstand a plausibility check. In particular discrepancies between the results of structural analysis (e.g. insufficient safety) and the real structural condition (e.g. no sign of distress or failure, satisfactory structural performance) must be explained. It should be kept in mind that many engineering models are conservative and cannot be always used directly to explain an actual situation.

The target reliability level used for verification can be taken as the level of reliability implied by acceptance criteria defined in proved and accepted design codes. 
The target reliability level can also be established taking into account the required performance level for the structure, the reference period and possible failure consequences. In accordance with ISO 2394 [2] the performance requirements for assessment of existing structures are the same as for design of a new structure. Lower reliability targets for existing structures may be used if justified on the basis of economical, social and sustainable considerations; more details are provided elsewhere [12, 13]. A simple model for specifying the target reliability of heritage structures was proposed in [14]. In accordance with Annex I of ISO 13822 [3] it is important to realise that the protection of heritage value may require the acceptance of a different reliability level, lower than that in design codes. Fundamental differences between structural design and the assessment of existing structures and protection of heritage values should be considered.

\section{Assessment in the case of damage}

For an assessment of a damaged structure the following stepwise procedure is recommended:

(1) Visual inspection: it is always useful to make an initial visual inspection of the structure to get a feel for its condition. Major defects should be reasonably evident to an experienced eye. In the case of very severe damage, immediate measures (like abandonment of the structure) may be taken.

(2) Explanation of observed phenomena: to be able to understand the present condition of the structure, one should simulate the damage or the observed behaviour, using a model of the structure and the estimated intensity of various loads or physical/chemical agencies. It is important to have available documentation with respect to design, analysis and construction. If there is a discrepancy between calculations and observations, it might be worthwhile to look for design errors, errors in construction etc.

(3) Reliability assessment: given the structure in its present state and given the present information, reliability of the structure is estimated either by means of a failure probability or by means of partial factors. The model of the present structure may be different from the original model. If reliability is sufficient (i.e. better than commonly accepted in design) one might be satisfied and no further action might be required.

(4) Additional information: if the reliability according to step 3 is insufficient, one may look for additional information from more advanced structural models, additional inspections and measurements or actual load assessment.

(5) Final decision: if the degree of reliability is still too low, one might decide to:

- accept the present situation for economical reasons;

- reduce the load on the structure (for instance traffic restrictions 
on road bridges);

- repair the structure;

- start demolition of the structure.

The first decision may be motivated by the fact that the cost of upgrading is much higher for a heritage structure than for a new structure. This argument is sometimes used by those who claim that a higher reliability should be generally required for a new structure than for an existing one. However, if human safety is involved, economical optimisation has a limited significance.

\section{Final report and decision}

The final report on structural assessment and possible interim reports (if required) should include clear conclusions with regard to the objective of the assessment based on careful reliability analysis and cost of repair or upgrading. The report shall be concise and clear; a recommended format is indicated in Annex G to ISO 13822 [3].

If an assessment shows insufficient reliability, appropriate interventions should be proposed. Temporary intervention may be recommended and proposed by an engineer if required immediately. The engineer should indicate a preferred solution as a logical follow-up to the whole assessment in every case.

It should be noted that the client in collaboration with a relevant authority should make the final decision on possible interventions, based on engineering assessment and recommendations. The engineer performing the assessment might have, however, the legal duty to inform the relevant authority if the client does not respond in a reasonable time.

Minimisation of construction interventions is required in rehabilitation and upgrades, but sufficient reliability should also be guaranteed. When dealing with the preservation of heritage structures, it may be difficult to propose construction interventions that respect all requirements for preservation of the heritage value. Based on the Venice Charter [15] principles of interventions seem to include:

- Safety of the construction;

- Respect of the heritage value (unobtrusiveness and respect of the original conception, minimum intervention, reversibility of the intervention, integration on the whole structure);

- Compatibility and durability of materials;

- Balance between costs and available financial resources;

- Indoor environment quality including aspects of comfort, security and accessibility.

Note that it is not always possible to follow all these principles as they may conflict with each other; for instance structural safety aspects often conflict with the protection of heritage values [1].

\section{Concluding remarks}

The main principles for assessment of heritage structures are: 
- Currently valid codes for verification of structural reliability should be applied, historic codes valid in the period when the structure was designed should be used only as guidance documents;

- Actual characteristics of structural materials, actions, geometric data and structural behaviour should be considered; original design documentation should be used as a guidance material only.

The most important step of the whole assessment procedure is evaluation of inspection data and updating of prior information concerning strength and structural reliability. It appears that a Bayesian approach can provide an effective tool. Typically, the assessment of the heritage structures is a cyclic process in which the first preliminary assessment is often supplemented by subsequent detailed investigations and analysis. A report on structural assessment prepared by an engineer should include a recommendation on possible intervention. However, the client in collaboration with the relevant authority should make the final decision concerning possible interventions.

\section{Acknowledgement}

This study is an outcome of the research project NAKI DF12P01OVV040 “Assessment of safety and working life of industrial heritage buildings".

\section{References}

[1] CIB, Guide for the Structural Rehabilitation of Heritage Buildings (CIB Publication 335), CIB: Rotterdam, pp. 48, 2010.

[2] ISO 2394, General principles on reliability for structures, pp. 73, 1998.

[3] ISO 13822, Bases for design of structures - Assessment of existing structures, pp. 44, 2010.

[4] ISO 12491, Statistical methods for quality control of building materials and components, pp. 30, 1997.

[5] Melchers, R.E., Structural Reliability Analysis and Prediction, John Wiley and Sons Ltd.: Chichester, England, pp. 437, 2001.

[6] Ellingwood, B.R., Reliability-based condition assessment and LRFD for existing structures. Structural Safety, 18(2-3), pp. 67-80, 1996.

[7] Lourenço, P.B., Recommendations for restoration of ancient buildings and the survival of a masonry chimney. Construction and Building Materials, 20(4), pp. 239-251, 2006.

[8] Sykora, M., Holicky, M. and Jung, K., Updating in the probabilistic assessment of existing structures. Transactions of the VSB - Technical University of Ostrava, Civil Engineering Series, XII(2), pp. 205-215, 2013.

[9] Ang, A.H.S. and Tang, W.H., Probabilistic Concepts in Engineering Emphasis on Applications to Civil and Environmental Engineering, John Wiley and Sons: USA, pp. 420, 2007. 
[10] Holicky, M., Reliability analysis for structural design, SUN MeDIA: Stellenbosch, pp. 199, 2009.

[11] Tanner, P., Lara, C. and Prieto, M., Semi-probabilistic models for the assessment of existing concrete structures. Proc. ICASP11, eds. M.H. Faber, J. Köhler and K. Nishijima, CRC Press/Balkema: Leiden, pp. 8, 2011.

[12] Sykora, M. and Holicky, M., Target reliability levels for the assessment of existing structures - case study. Proc. IALCCE 2012, eds. A. Strauss, K. Bergmeister and D.M. Frangopol, CRC Press: Leiden, pp. 813-820, 2012.

[13] Steenbergen, R.D.J.M. and Vrouwenvelder, A.C.W.M., Safety philosophy for existing structures and partial factors for traffic loads on bridges. Heron, 55(2), pp. 123-139, 2010.

[14] Schueremans, L. and Van Gemert, D., Assessing the safety of existing structures: reliability based assessment framework, examples and application. Journal of Civil Engineering and Management, X(2), pp. 131-141, 2004.

[15] ICOMOS, The Venice Charter 1964 (International Charter for the Conservation and Restoration of Monuments and Sites), International Council on Monuments and Sites: pp. 4, 1964. 